\title{
Transgenic Wheat Expressing a Barley UDP-Glucosyltransferase Detoxifies Deoxynivalenol and Provides High Levels of Resistance to Fusarium graminearum
}

\author{
Xin Li, ${ }^{1}$ Sanghyun Shin, ${ }^{2}$ Shane Heinen, ${ }^{2}$ Ruth Dill-Macky, ${ }^{3}$ Franz Berthiller, ${ }^{4}$ Natalya Nersesian, ${ }^{5}$ \\ Thomas Clemente, ${ }^{5}$ Susan McCormick, ${ }^{6}$ and Gary J. Muehlbauer ${ }^{1,2}$ \\ ${ }^{1}$ Department of Plant Biology, ${ }^{2}$ Department of Agronomy and Plant Genetics, and ${ }^{3}$ Department of Plant Pathology, University of \\ Minnesota, St. Paul, MN 55108, U.S.A.; ${ }^{4}$ Department of Agrobiotechnology (IFA-Tulln), University of Natural Resources and Life \\ Sciences, Vienna, 3430 Tulln, Austria; ${ }^{5}$ Department of Agronomy and Horticulture, University of Nebraska, Lincoln, NE 68588, \\ U.S.A.; ${ }^{6}$ USDA-ARS, Bacterial Foodborne Pathogen and Mycology Research Unit, Peoria, IL 61604, U.S.A.
}

Submitted 1 July 2015. Accepted 22 July 2015.

\begin{abstract}
Fusarium head blight (FHB), mainly caused by Fusarium graminearum, is a devastating disease of wheat that results in economic losses worldwide. During infection, $F$. graminearum produces trichothecene mycotoxins, including deoxynivalenol (DON), that increase fungal virulence and reduce grain quality. Transgenic wheat expressing a barley UDP-glucosyltransferase (HvUGT13248) were developed and evaluated for FHB resistance, DON accumulation, and the ability to metabolize DON to the less toxic DON-3-O-glucoside (D3G). Pointinoculation tests in the greenhouse showed that transgenic wheat carrying $\mathrm{HvUGT13248}$ exhibited significantly higher resistance to disease spread in the spike (type II resistance) compared with nontransformed controls. Two transgenic events displayed complete suppression of disease spread in the spikes. Expression of $\mathrm{HvUGT13248}$ in transgenic wheat rapidly and efficiently conjugated DON to D3G, suggesting that the enzymatic rate of DON detoxification translates to type II resistance. Under field conditions, FHB severity was variable; nonetheless, transgenic events showed significantly less-severe disease phenotypes compared with the nontransformed controls. In addition, a seedling assay demonstrated that the transformed plants had a higher tolerance to DON-inhibited root growth than nontransformed plants. These results demonstrate the utility of detoxifying DON as a FHB control strategy in wheat.
\end{abstract}

Fusarium head blight (FHB) (scab), primarily caused by Fusarium graminearum, is a disease of wheat and barley that results in dramatic losses of grain yield and quality (Goswami and Kistler 2004; Kazan et al. 2012; Parry et al. 1995). During

$\mathrm{X}$. Li and S. Shin contributed equally to this work.

Current address for S. Shin: National Institute of Crop Science (NICS), RDA, Suwon 441-857, Korea.

Corresponding author: G. J. Muehlbauer; Telephone: +1.612.625.6228; Fax: +1.612.625.1268; E-mail: mueh1003@umn.edu.

*The $\boldsymbol{e}$-Xtra logo stands for "electronic extra" and indicates that three supplementary figures and one supplementary table are published online.

(c) 2015 The American Phytopathological Society infection, F. graminearum produces trichothecene mycotoxins, including deoxynivalenol (DON), which facilitate disease development and contaminate grain (Goswami and Kistler 2004). Grain products contaminated with trichothecenes pose human and animal health issues when consumed (Pestka 2010; BinUmer et al. 2011). For this reason, the United States Food and Drug Administration has set the advisory level of DON at $1 \mathrm{ppm}$ $\left(\mathrm{mg} \mathrm{kg}^{-1}\right)$ in finished wheat products for human consumption (United States Food and Drug Administration 2010). Similarly, the European Union has established a maximum tolerance level of $0.75 \mathrm{ppm}$ for DON in food commodities for human consumption (European Commission 2006). Therefore, reducing trichothecene concentrations in grains is essential both for food safety and in disrupting FHB development.

The two major types of FHB resistance in wheat are recognized as type I (resistance to initial infection) and type II (resistance to disease spread in infected spikes) (Mesterhazy 1995). Numerous quantitative trait loci (QTL) associated with type I and II resistance have been identified (Anderson et al. 2001; Buerstmayr et al. 2009; Waldron et al. 1999). A major QTL for type II resistance was identified in wheat on the short arm of chromosome $3 \mathrm{~B}$ and is referred to as Fhbl (Anderson et al. 2001; Liu et al. 2006; Waldron et al. 1999). Intensive breeding efforts have been established that have resulted in incorporation of QTL into elite breeding lines; however, the level of resistance conferred by these QTL is insufficient to alone provide protection to $\mathrm{FHB}$, and the mechanisms of resistance provided by these QTL are not clear (Buerstmayr et al. 2009). Transgenic wheat events expressing host defense genes and DON-resistant genes have been developed, but these have only resulted in modest reductions in disease severity (Di et al. 2010; Mackintosh et al. 2007; Okubara et al. 2002; Shin et al. 2008). Although a recent report on host-induced gene silencing of a chitin synthase gene in transgenic wheat showed high levels of resistance to FHB (Cheng et al. 2015), there is a need to explore additional genetic mechanisms that perturb the infection process, thus resulting in improved FHB resistance.

Previous studies have shown that trichothecenes act as virulence factors during FHB disease development by contributing to disease spread in the infected spikes. F. graminearum strains carrying loss-of-function mutations in the TRI5 gene, which encodes the first enzyme in the trichothecene biosynthetic pathway, resulted in the lack of trichothecene production and reduced virulence on wheat (Proctor et al. 1995). Noteworthy, these trichothecene-deficient 
strains of $F$. graminearum can infect wheat but were unable to spread within the spike, demonstrating that trichothecenes are not required for initial infection but that they do play an important role in the spread of disease symptoms in the spike (Bai et al. 2002; Desjardins et al. 1996; Jansen et al. 2005; Proctor et al. 1997). Additional studies on the infection strategy of Fusarium spp. revealed that the pathogen has a biotrophic growth phase for about $48 \mathrm{~h}$ after spore germination. Subsequently, the pathogen produces trichothecenes and transitions to a necrotrophic strategy (Brown et al. 2010; Kazan et al. 2012; Lysøe et al. 2011). These findings indicate that suppression of trichothecene accumulation at early time points may be essential for limiting FHB development. Previous work by Lemmens et al. (2005) examined a population segregating for Fhbl QTL and found that lines containing the resistant Fhbl allele exhibited an increased ability to conjugate DON with glucose to the less-toxic DON-3-O-glucoside (D3G). These results indicated that the conjugation of DON to D3G is a potential resistance mechanism, and the authors proposed that Fhbl likely encodes for either a UDP-glucosyltransferase (UGT) or a gene that regulates UGT activity. Taken together, this information suggests that trichothecenes play a major role in pathogen virulence and the identification of appropriate genes that encode for UGTs for subsequent expression in transgenic plants may serve as an avenue to reduce DON accumulation and, in turn, disease severity.

Large gene families encode UGTs in plants (Caputi et al. 2012; Ross et al. 2001). These genes function by glucosylating plant metabolites and can also act to detoxify virulence factors produced by pathogens. An Arabidopsis UGT (DOGT1 or AtUGT73C5) was shown to conjugate DON at the carbon 3 position $(\mathrm{C} 3-\mathrm{OH})$ group with glucose to form D3G (Poppenberger et al. 2003). Overexpression of AtUGT73C5 in transgenic Arabidopsis resulted in DON resistance (Poppenberger et al. 2003). However, these same transgenic plants exhibited a dwarf phenotype, likely due to the conjugation of brassinosteroid brassinolide to the inactive brassinolide-23- $O$-glucoside (Poppenberger et al. 2005). A barley UGT, HvUGT13248, is upregulated by $F$. graminearum infection and DON application (Boddu et al. 2007; Gardiner et al. 2010). Functional characterization of HVUGT13248 showed that expression in both yeast and Arabidopsis imparts DON resistance by converting DON to D3G (Schweiger et al. 2010; Shin et al. 2012). In contrast to the transgenic Arabidopsis expressing DOGT1, Arabidopsis plants expressing HvUGT13248 did not exhibit a dwarf phenotype and the conjugation of brassinosteroids was not observed (Shin et al. 2012). Moreover, Brachypodium distachyon possesses two UGT homologs to HvUGT13248, which also showed resistance to high levels of DON by formation of D3G in yeast (Schweiger et al. 2013). These results suggest UGTs are promising genes for increased FHB resistance in wheat.

The overall goals of this study were to develop transgenic wheat expressing HvUGT13248 and to examine whether this gene increases resistance to $F$. graminearum infection and DON. The specific objectives of this study were i) to develop transgenic wheat constitutively expressing $H v U G T 13248$, ii) to evaluate these wheat lines for resistance to FHB in the greenhouse and in field trials, iii) to assess these wheat lines for resistance to DON, and iv) to monitor the conversion of DON to D3G in planta.

\section{RESULTS}

Generation of transgenic wheat expressing $H v U G T 13248$.

The plasmid, designated pZP212-HvUGT13248, carrying the $H v U G T 13248$ gene driven by the maize ubiquitin promoter, coupled with its first intron (Christensen et al. 1992) (Fig. 1A) was used for particle bombardment transformation of the wheat cv. Bobwhite (BW) and Agrobacterium-mediated transformation of the wheat cv. CBO37. The HvUGT13248 open reading frame has a C-terminal FLAG epitope, which enables
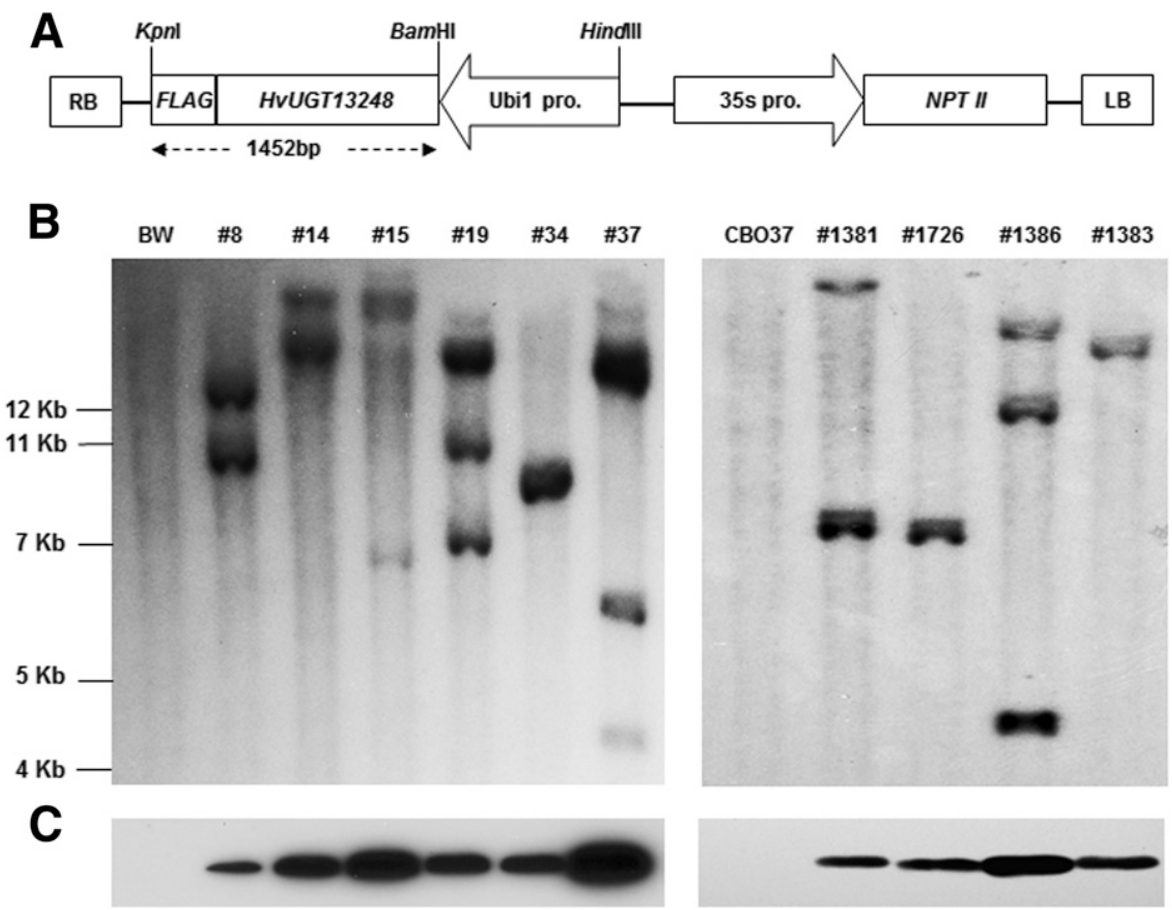

Fig. 1. Transformation plasmid and characterization of transgenic wheat. A, The pZP212 plasmid containing the HvUGT13248 transgene (pZP212HvUGT13248) was used for wheat transformation. NPT II = neomycin phosphotransferase II gene; Ubi-1 pro. = maize ubiquitin-1 promoter with the first intron; $35 \mathrm{~S}$ pro. $=$ Cauliflower mosaic virus $35 \mathrm{~S}$ promoter; $\mathrm{FLAG}=$ FLAG-epitope tag for Western blotting. B, Southern blot analysis using ${ }^{32} \mathrm{P}-\mathrm{dCTP}$ labeled NPTII gene and XbaI-digested genomic DNA. C, Western blot analysis using FLAG-epitope antibody. Events \#8, \#14, \#15, \#19, \#34, and \#37 were independent transgenic lines in the Bobwhite (BW) background and BW was the nontransformed control; \#1381, \#1726, \#1386, and \#1383 were independent transgenic lines in the $\mathrm{CBO} 37$ background and $\mathrm{CBO} 37$ was the nontransformed control. 
tracking of the $H v U G T 13248$ protein with FLAG-epitope antibodies. To this end, Western blot analysis using the FLAGepitope antibodies was conducted on the $T_{1}$ progeny derived from the primary transgenic events. Six BW transgenic events $(\# 8, \# 14, \# 15, \# 19, \# 34$, and \#37) and four CBO37 transgenic events (\#1381, \#1726, \#1386, and \#1383) accumulating detectable $H v U G T 13248$-FLAG protein levels were selected and were allowed to self-pollinate (Fig. 1C). Variation in expression levels of the transgene was observed across the selected events, with the nontransformed $\mathrm{BW}$ and $\mathrm{CBO} 37$ controls displaying no detectable signal (Fig. 1C). Coexpression of the HvUGT13248 protein with the NPTII selectable marker (enzyme-linked immunosorbent assay [ELISA] assay, described below) was observed. Thus, the NPTII ELISA assay was a convenient and reliable marker for the presence of the active $H v U G T 13248$ transgene and subsequent determinations of $H v U G T 13248$ transgene expression were based on the NPTII ELISA assay.

A Southern blot analysis was conducted on $\mathrm{T}_{3}$ individuals from the selected transgenic events to determine if they originated from independent transformation events. Genomic DNA was digested with the $X b a \mathrm{I}$ restriction endonuclease and was probed with the full-length NPTII gene. The probe did not hybridize to BW or CBO37 nontransformed controls, while each of the BW-derived and the CBO37-derived transgenic events exhibited different banding patterns, demonstrating that the transgene was successfully integrated into these lines and that they originated from independent transformation events (Fig. 1B).

\section{Evaluation of type II resistance conferred by transgenic wheat expressing HvUGT13248.}

To evaluate type II resistance in the transgenic wheat events, we performed point inoculation experiments under greenhouse conditions. For each screen, except for the Spring 2011 trial when there was limited seed availability, 20 plants of each transgenic event were grown and ELISA for the NPTII protein was performed on each plant. Between two to 20 plants from each transgenic event expressed the transgene, indicating that expression was relatively stable over generations (Supplementary Table S1). Plants expressing HvUGT13248 and, in some cases, the nonexpressing siblings (UGT-) were screened for FHB resistance. The susceptible check cv. Wheaton exhibited $84.7 \%$ FHB severity on average across all three trials, while the resistant check cv. Sumai 3 exhibited an average FHB severity of $10.1 \%$, indicating that the environment for disease progression was successful and discriminative. Our results show the transgenic events significantly reduced FHB severity compared with the nonexpressing siblings and nontransformed $\mathrm{BW}$ and $\mathrm{CBO} 37$ controls (Fig. 2). Compared with the nontransformed $\mathrm{BW}$ control, the reduction of disease severity across screening $T_{1}, T_{2}$, and $T_{3}$ plants from the $B W$ transgenic events was at least 74\%. Moreover, events \#15 and \#19 displayed a high degree of type II resistance in the greenhouse assays, with negligible fungal spread within the spike, an equivalent type II resistant phenotype to the Sumai 3 control. In a similar fashion, the combined results from the FHB greenhouse tests of the CBO37-derived transgenic events \#1381, \#1726, \#1386, and \#1383 displayed significant reduction in disease severity greater than $64 \%$ (Fig. 2), with CBO37 showing a more susceptible FHB phenotype than BW. Taken together, our results revealed that expression of $H v U G T 13248$ in wheat translates to enhanced type II resistance.

\section{Transgenic wheat expressing $\mathrm{HvUGT13248}$ promotes DON to D3G conversion.}

To determine if the expression of HvUGT13248 in transgenic wheat is catalyzing the conjugation of DON to D3G, hence contributing to the observed type II resistance, we monitored the rate of DON conjugation via quantification of DON and D3G over time. To this end, direct DON-challenge assays on transgenic event \#19 and the corresponding BW control were conducted. Plants from event \#19 were challenged with DON at a $2 \mu \mathrm{g}$ per spikelet rate applied to 25 spikelets $(50 \mu \mathrm{g}$ or $169 \mathrm{nmol}$ in total per spike) on the main spike per individual. Tissues samples were taken at 1, 3, 7, 14, and 21 days after DON challenge. DON and D3G levels were ascertained by liquid chromatography coupled to tandem mass spectrometry (LC-MS/MS). The conversion of DON to D3G was observed in event \#19 and the nontransformed BW. However, the conversion of DON to D3G was more rapid in event \#19 relative to $\mathrm{BW}$, with an average of $24 \%$ DON converted to D3G in event \#19 within the first $24 \mathrm{~h}$ after DON application, while there was
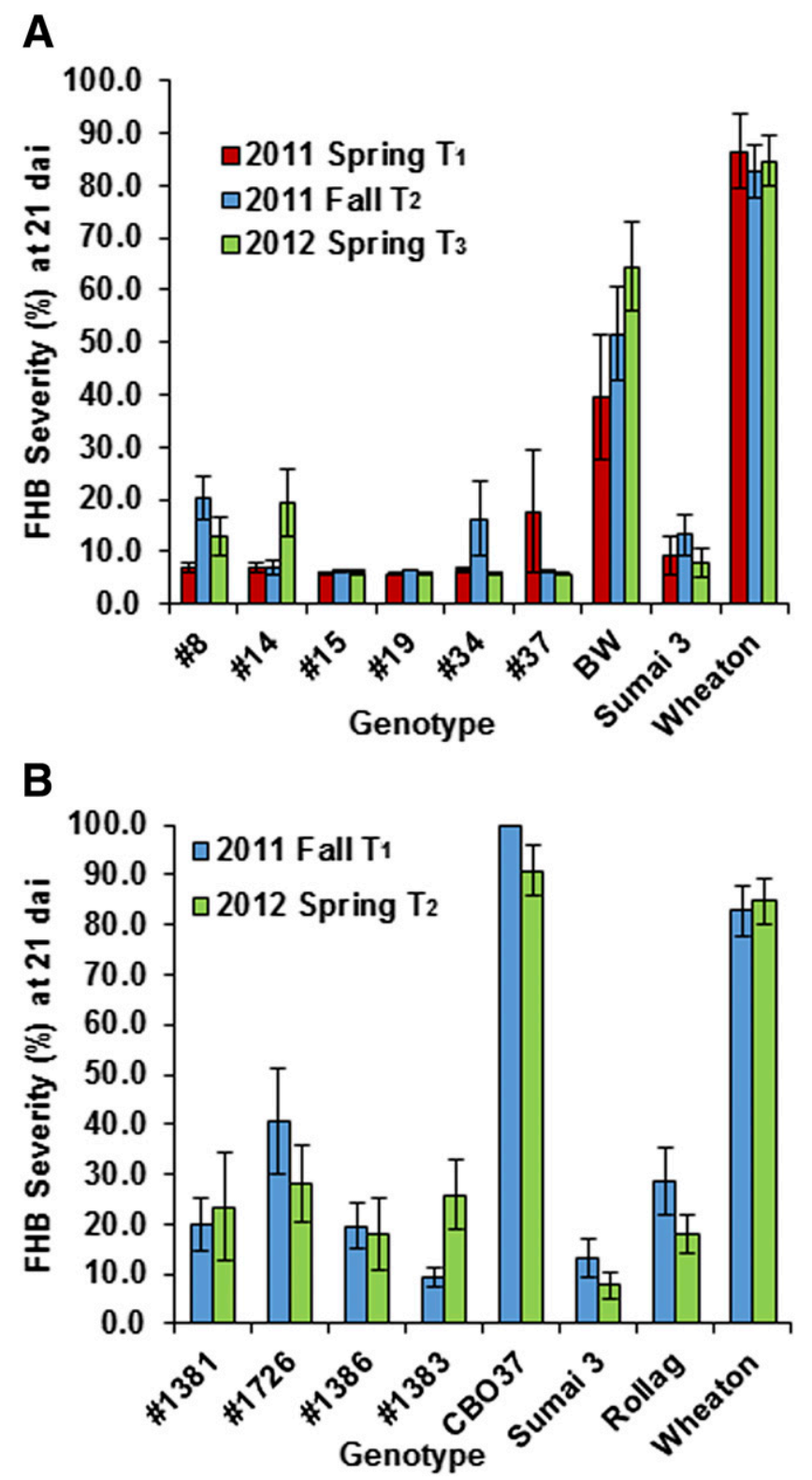

Fig. 2. Fusarium head blight (FHB) greenhouse screen of transgenic wheat expressing $H v U G T 13248$ at 21 days after point-inoculation. FHB severity was calculated as the percentage of symptomatic spikelets per spike at 21 days after inoculation. Wheaton was the susceptible check; Sumai 3 was the resistant check. A, Events \#8, \#14, \#15,\#19, \#34, and \#37 are transgenic wheat expressing $H v U G T 13248$ in the Bobwhite (BW) background; and B, events \#1381, \#1726, \#1386, and \#1383 are transgenic wheat expressing HvUGT13248 in the CBO37 background. 
only $2 \%$ DON conversion in BW. Between 1 and 3 days after DON application, the conversion of DON to D3G was greater in the nontransformed than transgenic plants, likely due to the lower level of DON in the transgenic plants. By 21 days after challenge, residual DON levels present in the transgenic plants were only $26 \%$ of that in BW (Fig. 3A and C). In addition, the D3G/DON ratio trended higher in event \#19 compared with BW (Fig. 3B). These results clearly demonstrate in planta UGT activity in the transgenic samples leads to the enhanced conversion of DON to D3G.

\section{Evaluation of field-based resistance conferred} by transgenic wheat expressing $H v U G T 13248$.

Transgenic wheat expressing HvUGT13248 exhibited a high level of type II resistance under greenhouse conditions. To gain insight on the translation of the resistance phenotype to a field environment, spray-inoculated experiments in mist-irrigated plots were conducted over the 2012 to 2014 growing seasons. Selected BW-derived transgenic lines were represented each year, while those from CBO37-derived transgenic lines were only in the 2013 and 2014 trials. Data on the various FHB-related phenotypes were monitored, including FHB incidence (percentage of spikes with visually symptomatic spikelets of the 20 heads observed), FHB severity (percentage of symptomatic spikelets of the total of all spikelets observed), percentage of visually scabby kernels (VSK), and DON concentration in harvested grain (Table 1). In addition, D3G concentration in grain from the 2013 and 2014 field trials was ascertained (Table 1).

Overall, FHB incidence, severity, and VSK in the BW- and CBO37-derived transgenic lines exhibited variable responses compared with the BW and CBO37 nontransformed controls, respectively. All transgenic lines exhibited a statistically significant reduction in FHB incidence and severity in at least one trial except for event \#1381 and VSK for at least one trial except for events \#14 and \#1381. Noteworthy, all BW-derived transgenic plants exhibited a statistically significant reduction in FHB severity in all three trials except for event \#37 in 2014. Importantly, event \#37 in 2012 and 2013 and event \#1726 in 2013 and 2014 exhibited similar levels of FHB severity and VSK compared with the type II resistant commercial varieties Rollag and Alsen that carry the Fhbl QTL. It is also worth noting that most transgenic events showed significantly reduced FHB incidence except in 2014. Taken together, our results demonstrate that transgenic wheat expressing $H v U G T 13248$ can reduce the level of FHB severity that is comparable to FHBresistant commercial varieties and the FHB incidence and VSK can be reduced when FHB disease pressure is moderate (e.g., 2013).
A

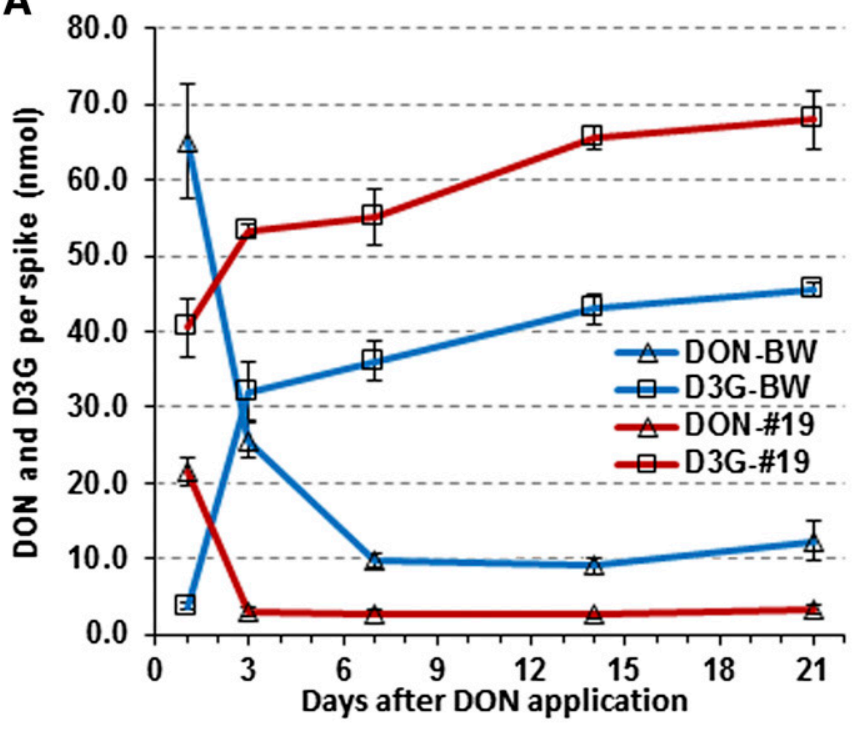

B

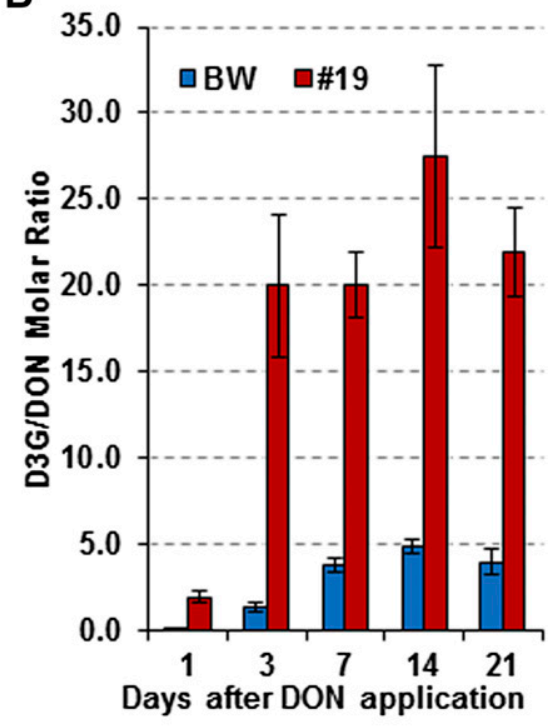

C

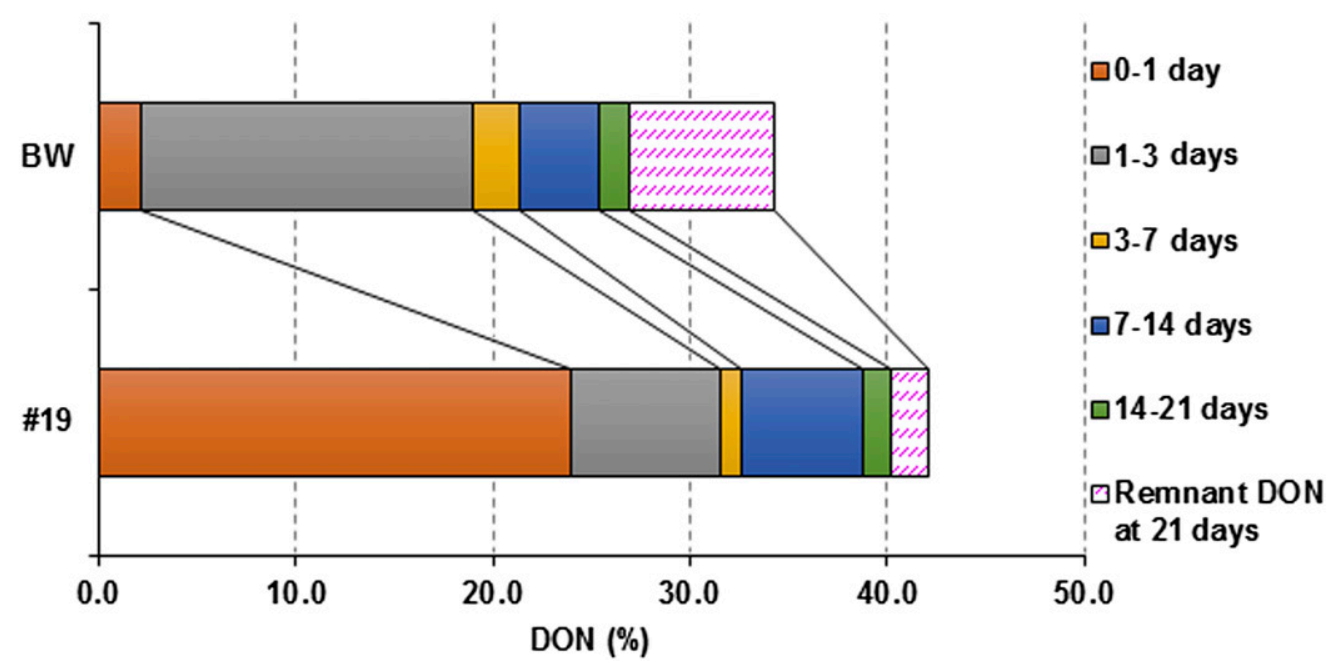

Fig. 3. Conjugation of deoxynivalenol (DON) to DON-3-O-glucoside (D3G) in transgenic wheat expressing $H v U G T 13248$. A, DON and D3G concentrations in Bobwhite (BW) and transgenic line \#19 at each time point, from 1 to 21 days after DON application. B, Fold change of molar ratio of D3G to DON concentrations in BW and transgenic line \#19 at each time point. C, Rate of DON converted to D3G over time. 
Since $H v U G T 13248$ conjugates DON to D3G, the expected outcome is a lower concentration of DON, a higher concentration of $\mathrm{D} 3 \mathrm{G}$, and a higher ratio of $\mathrm{D} 3 \mathrm{G} / \mathrm{DON}$ in the transgenic plants compared with the nontransformed controls. All transgenic lines except \#19 showed a higher ratio of D3G/DON than nontransformed controls in at least one trial; however, we observed several possible combinations of DON and D3G concentration in the transgenic plantss compared with the nontransformed controls. Consistent with our initial prediction for just the DON and D3G concentrations were lines \#14 and \#1386 in 2013, which exhibited statistically significant decreases in DON concentration and significant increases in D3G concentration in the transgenic plants compared with the nontransformed controls. We also observed significant decreases in DON and nonsignificant changes in D3G concentration (e.g., lines \#8, \#15, and \#19 in 2013; lines \#1726 and \#1383 in 2014), significant decreases in both DON and D3G (e.g., line \#1726 in 2013), nonsignificant changes in DON concentration and significant increases in D3G concentration (e.g., lines \# 8, \#14, $\# 15$, and \#37 in 2014), and nonsignificant changes in DON and D3G concentration (e.g., \#1383 in 2013; \#19 in 2014, \#1381 in 2013 and 2014). These results show that HvUGT13248 conjugates DON to D3G in field conditions but that there are considerable environmental factors that play a role in the DON and D3G concentrations in the grain.

The three field trials provided the opportunity to assess the protection $H v U G T 13248$ offered in environments exhibiting different levels of FHB pressure and to assess the relationship between the ratio of DON and D3G concentration and disease severity. We judged the severity of each field trial based on the level of FHB incidence in the check lines included each year. In the low disease environment in 2013, the transgenic plants exhibited the lowest level of incidence, severity, and VSK. Noteworthy, in 2013, the DON concentrations of all the transgenic plants were lower than the controls, and, in the BWderived transgenic events, the DON and D3G combined levels

Table 1. Field screening results of transgenic wheat expressing HvUGT13248

\begin{tabular}{|c|c|c|c|c|c|c|c|}
\hline Line & Incidence $^{b}$ & Severity $^{c}$ & $\operatorname{VSK}^{d}$ & $D_{O N}{ }^{e}$ & D3G $^{\mathrm{e}}$ & D3G/DON & $\mathrm{DBG}^{-\mathrm{DON}^{\mathrm{e}}}$ \\
\hline \multicolumn{8}{|l|}{2012} \\
\hline BW & $96 \pm 2$ & $40 \pm 4$ & $26 \pm 5$ & $38 \pm 5$ & NA & NA & NA \\
\hline \#8 & $76 \pm 7^{*}$ & $15 \pm 8 * *$ & $18 \pm 1$ & $55 \pm 4^{*}$ & NA & NA & NA \\
\hline$\# 14$ & $89 \pm 8$ & $24 \pm 2 * *$ & $20 \pm 4$ & $40 \pm 8$ & NA & NA & NA \\
\hline \#15 & $72 \pm 6^{* *}$ & $10 \pm 2 * * *$ & $9 \pm 1 * *$ & $27 \pm 5$ & NA & NA & NA \\
\hline \#19 & $65 \pm 9 * *$ & $11 \pm 2 * * *$ & $14 \pm 1 *$ & $28 \pm 2$ & NA & NA & NA \\
\hline \#37 & $56 \pm 9 * *$ & $7 \pm 2 * * *$ & $9 \pm 2 * * *$ & $26 \pm 2^{*}$ & NA & NA & NA \\
\hline Rollag & $91 \pm 5$ & $27 \pm 3$ & $16 \pm 3$ & $18 \pm 3$ & NA & NA & NA \\
\hline Sumai 3 & $48 \pm 1$ & $4 \pm 0$ & $4 \pm 1$ & $8 \pm 3$ & NA & NA & NA \\
\hline Alsen & $90 \pm 5$ & $25 \pm 3$ & $16 \pm 2$ & $3 \pm 7$ & NA & NA & NA \\
\hline Wheaton & $98 \pm 1$ & $42 \pm 7$ & $75 \pm 0$ & $59 \pm 12$ & NA & NA & NA \\
\hline \multicolumn{8}{|l|}{2013} \\
\hline BW & $91 \pm 4$ & $27 \pm 5$ & $8 \pm 1$ & $26 \pm 3$ & $8 \pm 1$ & $0.30 \pm 0.02$ & $34 \pm 4$ \\
\hline \#8 & $74 \pm 6^{*}$ & $11 \pm 2 *$ & $5 \pm 1 * *$ & $12 \pm 2 * *$ & $15 \pm 4$ & $1.16 \pm 0.23^{*}$ & $27 \pm 6$ \\
\hline \#14 & $81 \pm 2 *$ & $12 \pm 1 *$ & $7 \pm 1$ & $18 \pm 2^{*}$ & $17 \pm 1 * *$ & $0.94 \pm 0.13 * *$ & $35 \pm 2$ \\
\hline \#15 & $68 \pm 6^{*}$ & $9 \pm 1 * *$ & $3 \pm 1 * * *$ & $8 \pm 1 * * *$ & $8 \pm 3$ & $0.95 \pm 0.34$ & $16 \pm 4^{*}$ \\
\hline \#19 & $63 \pm 4 * *$ & $11 \pm 2 *$ & $5 \pm 0 * *$ & $11 \pm 3 * *$ & $7 \pm 3$ & $0.52 \pm 0.14$ & $18 \pm 6$ \\
\hline \#37 & $39 \pm 8 * * *$ & $4 \pm 1 * *$ & $4 \pm 1 * *$ & $11 \pm 2 * *$ & $13 \pm 5$ & $1.06 \pm 0.31$ & $24 \pm 6$ \\
\hline CBO37 & $83 \pm 3$ & $17 \pm 3$ & $19 \pm 1$ & $39 \pm 3$ & $22 \pm 5$ & $0.57 \pm 0.12$ & $61 \pm 6$ \\
\hline \#1381 & $76 \pm 7$ & $15 \pm 4$ & $16 \pm 1$ & $30 \pm 4$ & $35 \pm 7$ & $1.13 \pm 0.11 *$ & $65 \pm 11$ \\
\hline \#1726 & $44 \pm 9 * *$ & $5 \pm 1 * *$ & $4 \pm 1 * * *$ & $8 \pm 1 * * *$ & $6 \pm 1^{*}$ & $0.71 \pm 0.09$ & $14 \pm 2 * * *$ \\
\hline \#1386 & $55 \pm 5^{* *}$ & $7 \pm 1 * *$ & $11 \pm 2 * *$ & $27 \pm 4 *$ & $56 \pm 9 * *$ & $2.07 \pm 0.12 * * *$ & $83 \pm 13$ \\
\hline \#1383 & $74 \pm 4$ & $9 \pm 2 *$ & $8 \pm 2 * * *$ & $28 \pm 5$ & $33 \pm 7$ & $1.16 \pm 0.08 * *$ & $61 \pm 11$ \\
\hline Rollag & $63 \pm 11$ & $7 \pm 1$ & $5 \pm 1$ & $10 \pm 1$ & $3 \pm 0$ & $0.35 \pm 0.05$ & $14 \pm 0$ \\
\hline Sumai 3 & $44 \pm 3$ & $3 \pm 0$ & $2 \pm 1$ & $7 \pm 2$ & $2 \pm 1$ & $0.46 \pm 0.21$ & $10 \pm 3$ \\
\hline Alsen & $75 \pm 5$ & $11 \pm 1$ & $5 \pm 1$ & $8 \pm 1$ & $2 \pm 1$ & $0.29 \pm 0.10$ & $10 \pm 1$ \\
\hline Wheaton & $95 \pm 3$ & $34 \pm 4$ & $43 \pm 1$ & $29 \pm 5$ & $7 \pm 2$ & $0.22 \pm 0.04$ & $35 \pm 7$ \\
\hline \multicolumn{8}{|l|}{2014} \\
\hline BW & $100 \pm 0$ & $64 \pm 3$ & $22 \pm 2$ & $74 \pm 16$ & $6 \pm 1$ & $0.09 \pm 0.02$ & $81 \pm 17$ \\
\hline \#8 & $100 \pm 0$ & $33 \pm 3 * * *$ & $18 \pm 2$ & $107 \pm 16$ & $21 \pm 1 * * *$ & $0.22 \pm 0.04 *$ & $128 \pm 16$ \\
\hline \#14 & $98 \pm 3$ & $42 \pm 6^{* *}$ & $21 \pm 2$ & $95 \pm 14$ & $21 \pm 3 * *$ & $0.23 \pm 0.03 * *$ & $115 \pm 16$ \\
\hline$\# 15$ & $96 \pm 2$ & $37 \pm 11 *$ & $21 \pm 3$ & $75 \pm 12$ & $16 \pm 2 * *$ & $0.22 \pm 0.02 * *$ & $91 \pm 14$ \\
\hline \#19 & $100 \pm 0$ & $47 \pm 4 * *$ & $24 \pm 4$ & $77 \pm 10$ & $10 \pm 1$ & $0.13 \pm 0.01$ & $87 \pm 11$ \\
\hline \#37 & $99 \pm 1$ & $57 \pm 4$ & $20 \pm 3$ & $64 \pm 9$ & $19 \pm 2 * *$ & $0.30 \pm 0.02 * * *$ & $83 \pm 12$ \\
\hline $\mathrm{CBO} 37$ & $98 \pm 1$ & $34 \pm 5$ & $25 \pm 3$ & $111 \pm 8$ & $17 \pm 2$ & $0.16 \pm 0.02$ & $128 \pm 9$ \\
\hline \#1381 & $91 \pm 3$ & $25 \pm 4$ & $19 \pm 1$ & $84 \pm 12$ & $20 \pm 3$ & $0.23 \pm 0.01 * *$ & $104 \pm 14$ \\
\hline \#1726 & $84 \pm 7$ & $16 \pm 2 * *$ & $16 \pm 3^{*}$ & $52 \pm 8 * *$ & $18 \pm 1$ & $0.38 \pm 0.08 *$ & $70 \pm 7 * *$ \\
\hline \#1386 & $95 \pm 2$ & $32 \pm 3$ & $20 \pm 1$ & $98 \pm 21$ & $25 \pm 1^{*}$ & $0.28 \pm 0.05$ & $123 \pm 20$ \\
\hline \#1383 & $81 \pm 6^{*}$ & $13 \pm 1 * *$ & $8 \pm 1 * *$ & $57 \pm 6^{* *}$ & $16 \pm 1$ & $0.28 \pm 0.03^{* *}$ & $73 \pm 7 * *$ \\
\hline Rollag & $93 \pm 1$ & $23 \pm 1$ & $15 \pm 2$ & $32 \pm 8$ & $2 \pm 0$ & $0.07 \pm 0.01$ & $34 \pm 8$ \\
\hline Sumai 3 & $91 \pm 2$ & $18 \pm 2$ & $9 \pm 2$ & $22 \pm 4$ & $3 \pm 0$ & $0.13 \pm 0.02$ & $25 \pm 4$ \\
\hline Alsen & $93 \pm 3$ & $29 \pm 3$ & $18 \pm 4$ & $42 \pm 9$ & $4 \pm 0$ & $0.10 \pm 0.03$ & $46 \pm 9$ \\
\hline Wheaton & $98 \pm 3$ & $72 \pm 9$ & $75 \pm 0$ & $107 \pm 21$ & $6 \pm 0$ & $0.07 \pm 0.01$ & $113 \pm 21$ \\
\hline
\end{tabular}

a Events \#8, \#14, \#15, \#19 and \#37 are transgenic wheat expressing HvUGT13248 in the Bobwhite (BW) background, and events \#1381, \#1726, \#1386 and \#1383 are expressing HvUGT13248 in the CBO37 background. Wheaton was the susceptible check; Alsen, Rollag and Sumai 3 were type II resistant checks; BW and CBO37 were the nontransformed controls. Values provided are the means \pm standard error. *, ** and $* * *$ indicate significance at the $0.05,0.01$ and 0.001 levels compared with the BW or CBO37 nontransformed controls. NA = not available.

b Fusarium head blight (FHB) incidence, the percentage of spikes with visually symptomatic spikelets of the 20 heads observed.

c FHB severity, the percentage of diseased spikelets of the total of the 20 spikes observed.

${ }^{\mathrm{d}}$ VSK (\%): the percentage of visually scabby kernels (VSK).

e Deoxynivalenol (DON), DON-3-O-glucoside (D3G), and D3G+DON concentrations are shown in nanomoles per gram. 
were lower than those of the nontransformed BW lines, except for transgenic event \#14. In the higher disease environment in 2012, we observed a trend toward a significant reduction in FHB incidence, FHB severity, and VSK, but the DON concentration in the transgenic plants compared with the control was not significantly different. In the highest disease environment in 2014, we observed a significant decrease in FHB severity of the transgenic plants, although the other disease parameters exhibited high variation. Only transgenic line \#1726 showed consistently high levels of resistance across the disease environments, with lower FHB incidence, a higher D3G/DON ratio, and statistically significantly lower FHB severity, VSK, $\mathrm{DON}$, and DON and D3G combined than the nontransformed CBO37. Our results indicate a general trend for the transgenic plants to perform best in an environment with reduced disease pressure, although, even under high disease pressure, the transgenic plants can confer a significant reduction in FHB incidence, FHB severity, and VSK, but considerable variability with regards to DON and D3G concentration is apparent.
Transgenic wheat expressing HvUGT13248 provides resistance in roots to DON.

DON is known to inhibit root elongation in Arabidopsis and wheat (Masuda et al. 2007), so we examined the root growth of selected HvUGT13248 events on DON-supplemented growth medium (Fig. 4, Supplementary Figs. S1 and S2). $\mathrm{T}_{4}$ seeds of homozygous lineages derived from transgenic events $\# 8$, \#15, and \#37 along with the corresponding control BW were germinated on Murashige and Skoog (MS) medium for $24 \mathrm{~h}$ and were subsequently transferred to MS medium supplemented with $0,2,5$, and $10 \mathrm{ppm}$ (milligrams per liter) DON. Root growth was measured once a day from 3 to 7 days after germination. In control treatments $(0 \mathrm{ppm}$ DON), rootgrowth measurements for the transgenic events were not significantly different from BW (Fig. 4). However, in plates containing DON, root growth from the transgenic events was significantly longer than BW (Fig. 4). Similar results were observed in the transgenic events in the $\mathrm{CBO} 37$ background.
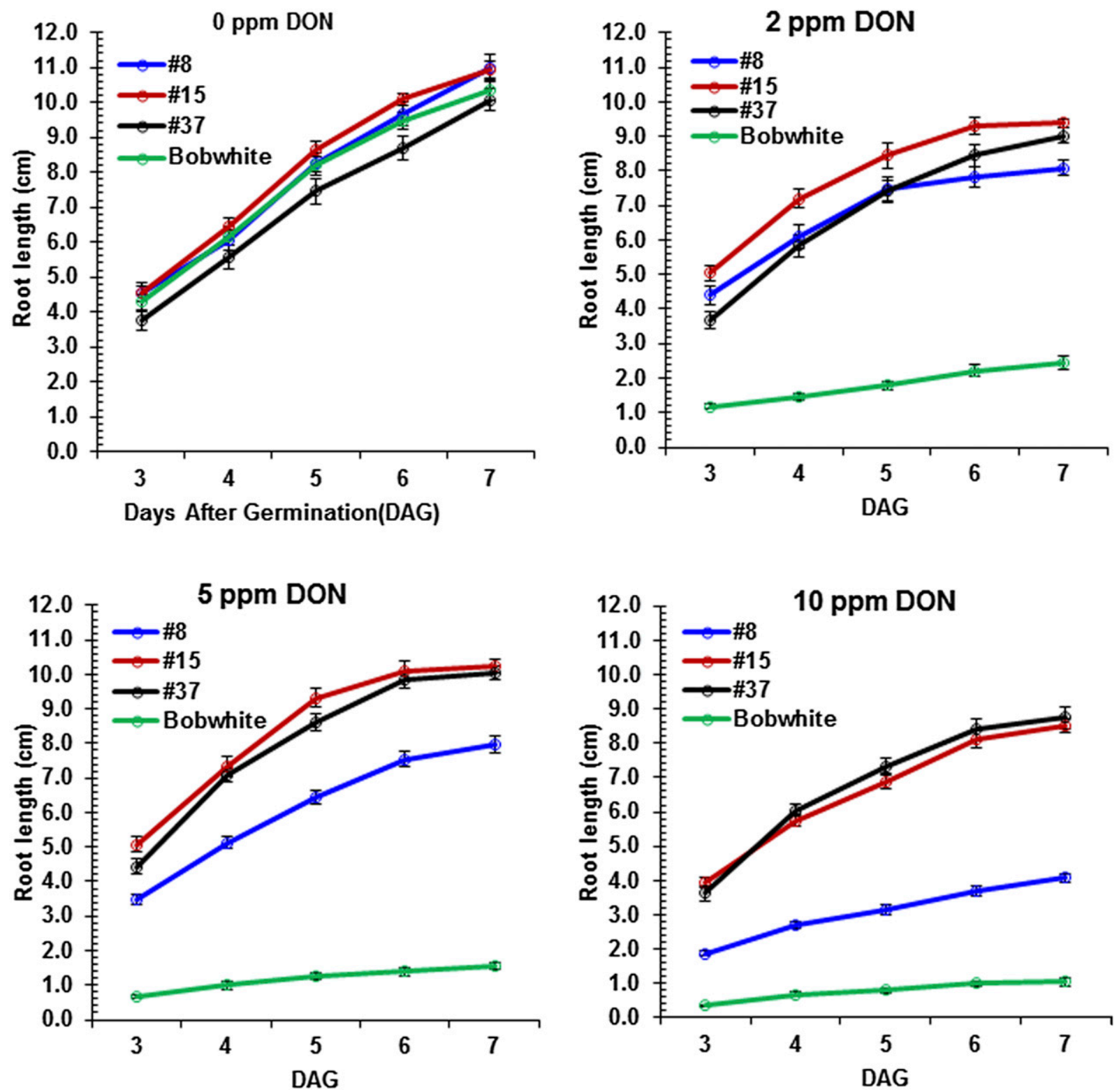

Fig. 4. Root growth of transgenic wheat lines (\#8, \#15, and \#37) in the Bobwhite background expressing HvUGT13248 on Murashige and Skoog medium containing $0,2,5$, and 10 ppm deoxynivalenol (DON). 


\section{Expression of $\mathrm{HvUGT13248}$}

in wheat does not alter morphology.

To determine if the expression of HvUGT13248 in wheat resulted in morphological changes compared with the controls, we measured the height of the transgenic wheat plants and the controls in field trials in 2013 and 2014. Our results showed that the transgenic plants were indistinguishable, except for \#1726 in 2013, from the controls under both field environments (Supplementary Fig. S3).

\section{DISCUSSION}

Transgenic wheat expressing HvUGT13248 exhibit FHB resistance and does not alter morphology.

Plant UGTs have previously been demonstrated to conjugate DON to the less toxic metabolite D3G in both yeast (Poppenberger et al. 2003; Schweiger et al. 2010, 2013) and Arabidopsis (Poppenberger et al. 2003; Shin et al. 2012) systems. To address the potential to translate these findings from model systems to commodity crops as a means to mitigate pathogenesis of Fusarium spp., we introduced $H v U G T 13248$ into two wheat genetic backgrounds, BW and CBO37, and subsequently phenotyped a set of derived transgenic events under laboratory, greenhouse, and field-based environments. The data tabulated from these studies clearly show that transgenic wheat events expressing HvUGT13248 had significantly lower FHB severities compared with their respective controls, over diversified environmental conditions and across two genetic backgrounds (Table 1; Fig. 2). The results herein reveal HvUGT13248 expression in wheat can lead to an equivalently high level of type II resistance to the type II-resistant cv. Sumai 3 (Fig. 2). Previous transgenic approaches to enhance FHB resistance in wheat included the introduction of antifungal proteins wherein reduction in disease severity was modest in the greenhouse, ranging from 30 to 52\% (Di et al. 2010; Mackintosh et al. 2007; Shin et al. 2008). Importantly, HvUGT13248 not only enhances the type II resistance response but also led to significant reduction in FHB incidence, except in 2014, when disease pressure was high, which indicates that $H v U G T 13248$ may also contribute to the type I resistance response by detoxifying DON at an early stage of initial infection. Interestingly, trichothecene biosynthesis is induced during the establishment of initial infection (Boenisch and Schäfer, 2011) but not needed for initial infection (Bai et al. 2002). Thus, additional research is required to determine the role that conjugation of DON to D3G may play in resistance to initial infection.

$H v U G T 13248$ belongs to the glycosyltransferase gene superfamily, which includes a plethora of homologous genes in plants, including Arabidopsis thaliana, Brachypodium distachyon, wheat, and barley (Caputi et al. 2012; Li et al. 2001; Schweiger et al. 2010, 2013). The Arabidopsis DOGT1 gene is capable of conjugating DON to $\mathrm{D} 3 \mathrm{G}$, and overexpression of DOGT1 in Arabidopsis resulted in increased tolerance against DON and 15ADON (15-acetylated DON) (Poppenberger et al. 2003). However, DOGT1 also catalyzes glucoside conjugation with brassinosteroids, resulting in a dwarf phenotype in transgenic Arabidopsis plants (Poppenberger et al. 2005). Unlike DOGT1, HvUGT13248 does not produce brasinosteroid glucosides in Arabidopsis plants, and the morphology of the transgenic Arabidopsis was similar to the nontransformed controls (Shin et al. 2012). Consistent with the previous results in Arabidopsis, our results showed that expression of HvUGT13248 in wheat, with the exception of \#1726 in 2013, does not alter plant height.

\section{Rapid and large amount of conjugation} of DON to D3G results in type II resistance.

The underlying mechanism associated with the observed enhanced type II resistance imparted by expression of the
$H v U G T 13248$ in wheat is enzymatic activity leading to the increased conversion rate of DON to D3G (Fig. 3). Although DON glycosylation is a common detoxification strategy in cereal crops and D3G is identified in naturally contaminated fields (Berthiller et al. 2005, 2009), our results show that the rate and amount of conjugation, especially at the early stage of infection, is important for resistance. In our feeding experiment, BW converted DON to D3G; however, transgenic line \#19 converted a larger portion of DON to D3G in the first $24 \mathrm{~h}$. In our field tests, both nontransformed controls BW and CBO37 accumulated higher levels of D3G compared with resistant checks (Sumai 3, Rollag, and Alsen), and even the susceptible check Wheaton showed the ability to convert DON to D3G. However, in all these lines, the ratio of $\mathrm{D} 3 \mathrm{G} / \mathrm{DON}$ is much lower than all of the transgenic lines. These results are consistent with previous reports of transgenic yeast and Arabidopsis expressing HvUGT13248 that showed higher resistance to DON in the growth media and, ultimately, higher ratios of D3G/DON compared with the nontransformed controls (Schweiger et al. 2010; Shin et al. 2012).

Previous attempts to provide FHB resistance through trichothecene metabolism via a detoxification route were largely ineffective. The Tri101 gene encodes an enzyme that catalyzes the acetylation of the hydroxyl group at the C3-OH of trichothecene precursors to reduce toxicity (McCormick et al. 1999). Transgenic tobacco plants expressing the $F$. sporotrichioidesderived Tril01 gene (FsTri101) showed increased tolerance to trichothecenes (Muhitch et al. 2000). However, when FsTri101 was overexpressed in transgenic wheat (Okubara et al. 2002), the derived transgenic plants only showed partial type II resistance after point inoculation under greenhouse conditions.

D3G is considered a 'masked' trichothecene (Berthiller et al. 2005), given that $D 3 G$ is not monitored during routine assays for trichothecenes but can be converted back to DON by the gut microflora of mammals (Berthiller et al. 2011). Thus, although D3G is less phytotoxic than DON (Poppenberger et al. 2003), the accumulation of $\mathrm{D} 3 \mathrm{G}$ in grain is still considered a potential health issue. However, the rapid conjugation of DON to D3G during the early stages of infection may reduce overall fungal load, which in turn may result in reduced DON and D3G in grain. For example, the most resistant transgenic events, \#37 and \#1726, both accumulated lower total toxin levels (DON+D3G) in the 2013 field trial, as compared with the corresponding nontransformed controls (Table 1).

In summary, our results show that transgenic wheat expressing $H v U G T 13248$ is an intriguing option for further exploration with the goal to detoxify DON and control FHB.

\section{MATERIALS AND METHODS}

\section{Plant materials.}

The spring wheat cultivars Alsen, Sumai 3, Rollag, Wheaton, $\mathrm{BW}$, and $\mathrm{CBO} 37$ were used for the experiments. BW and $\mathrm{CBO} 37$ were used for transformation experiments. Wheaton and $\mathrm{CBO} 37$ are spring wheat cultivars that are highly susceptible to FHB. $\mathrm{BW}$ is a spring wheat cultivar that exhibits moderate susceptibility. Alsen and Rollag are spring wheat cultivars carrying the Fhb1 QTL inherited from Sumai 3 and exhibit type II resistance and are moderately resistant. Sumai 3 is a Chinese cultivar that exhibits high levels of type II FHB resistance.

\section{Plant transformation vector and wheat transformation.}

The 1,452-bp barley UDP-glucosyltransferase (HvUGT13248) gene containing a carboxyl-terminal FLAG-tag sequence was cloned into the pENTER TM/D TOPO vector (Shin et al. 2012) and was then inserted into the binary expression plasmid pIPKb002 (Himmelbach et al. 2007), using the Gateway LR recombination 
reaction. The Ubi1 promoter forward 5'-GGGAAGCTTGGCCT TACTAGGCTGCAGTG-3' and nopaline synthase (NOS) terminator reverse 5'-CCCGGTACCCGCGTCGAGCGATCTAGTA-3' primers were used for the $H v U G T 13248$ polymerase chain reaction (PCR) amplification including the Ubil promoter and NOS terminator. The $2.9-\mathrm{kb}$ PCR product was digested and cloned into the unique HindIII and $K p n I$ sites of the vector pZP212. The pZP212 plasmid contains the NPTII gene driven by the Cauliflower mosaic virus $35 \mathrm{~S}$ promoter and terminated by the NOS gene from Agrobacterium tumefaciens (Hajdukiewicz et al. 1994). The spring wheat cultivar BW was used for transformation by particle gun bombardment method. The transformation protocols, including particle gun bombardment of embryos, tissue culture selection, and plant regeneration, were conducted according to the protocol described by Mackintosh and colleagues (2006). The CBO37-derived transgenic lines were produced using Agrobacterium-mediated transformation according to Cheng and colleagues (1997).

\section{Characterization of transgenic wheat expressing $\mathrm{HvUGT13248.}$}

For Southern blot analysis of transgenic wheat carrying the $H v U G T 13248$ gene, genomic DNA $(10 \mu \mathrm{g})$ was digested with endonuclease $X b a \mathrm{I}$, was separated on a $1 \%$ agarose gel, and was transferred onto Hybond $\mathrm{N}^{+}$membranes (Amersham Biosciences, Piscataway, NJ, U.S.A.). The NPTII gene probe (795 bp) was derived from a PCR-amplified product. The forward 5'-ATGATTGAACAAGATGGATTG-3' and reverse 5'TCAGAAGAACTCGTCAAG-3' ${ }^{\prime}$ primers were used for the NPTII gene probe PCR amplification. The probe sequence was labeled with ${ }^{32} \mathrm{P}$ dCTP using the Prime-a-Gene labeling system (Promega, Madison, WI, U.S.A.), following the manufacturer's instructions. The radiolabeled NPTII gene was used as a probe for the hybridization, and the subsequent banding patterns were visualized using autoradiography.

For Western blot analysis, protein was extracted by grinding leaf tissues in extraction buffer $(2 \%$ sodium dodecyl sulfate [SDS], $60 \mathrm{mM}$ Tris- $\mathrm{HCl}$ [pH 6.8], $14.4 \mathrm{mM} \beta$-mercaptoethanol, $10 \%$ glycerol, and $0.1 \%$ [wt/vol] bromophenol blue) and cell debris was removed by centrifugation. Total protein concentration was determined using Bio-Rad reagent (Bio-Rad, Hercules, CA, U.S.A.), with bovine serum albumin as a standard. Protein extracts (10 $\mu \mathrm{g}$ for BW transgenic lines and $15 \mu \mathrm{g}$ for CBO37 lines) were separated by SDS-polyacrylamide electrophoresis (12\% acrylamide) and were transferred to polyvinylidene diflouride transfer membrane (Amersham Biosciences, Piscataway, NJ, U.S. A.). A DYKDDDK tag horseradish peroxidase-conjugated antibody recognizing the FLAG-tag sequence (Cell Signaling Technology, Beverly, MA, U.S.A.) was used to detect the HvUGT13248-Flag protein at a 1:3,000 dilution. The protein was visualized using the SuperSignal West Pico chemiluminescent substrate (Thermo Scientific, Pierce Biotechnology, Rockford, IL, U.S.A.).

\section{Greenhouse screening \\ of transgenic lines against $F$. graminearum.}

Plants were sown into Sunshine MVP growth medium (Sun Gro Horticulture, Agawam, MA, U.S.A.) in 6-inch square plastic pots in a greenhouse. Seeds from each transgenic line were planted four seeds per pot. Nontransformed controls (BW, CBO37, Sumai 3, and Wheaton) were also planted four seeds per pot. Plants were fertilized with 1 teaspoon of Osmocote (14-14-14 N-P-K; Scotts Company, Marysville, OH, U.S.A.) fertilizer per pot at the threeleaf stage. ELISA kits (Agdia Inc., Elkhart, IN, U.S.A.) for the NPTII antibody were used to identify plants expressing the transgene. At anthesis, one floret of a central spikelet of the main spike was inoculated with $10 \mu \mathrm{l}$ of $F$. graminearum macroconidial suspension $\left(10^{5}\right.$ macroconidia per milliliter). Inoculated spikes were covered with transparent plastic bags for 3 days. FHB disease severity was determined as the percentage of spikelets with disease symptoms on the inoculated spikes at 21 days after inoculation (dai). For statistical analysis, Student's $t$ tests were used to compare each transgenic event to the parental nontransformed controls. All analysis was performed with Microsoft Excel 2013 (Microsoft Corp., Redmond, WA, U.S.A.).

\section{Conversion of DON to D3G in planta.}

For transgenic event \#19 in the BW background and the nontransformed BW control, $10 \mu \mathrm{l}$ of DON $\left(0.2 \mu \mathrm{g} \mu \mathrm{l}^{-1}\right)$ was introduced, at anthesis between the palea and lemma, in each of 25 florets on the main spike of each plant. In this manner, each spike received $50 \mu \mathrm{g}$ of DON. Three spikes of each genotype were bulksampled at 1, 3, 7, 14, and 21 dai. Three biological replications were conducted for each time point for each genotype. The spikes for each replication were ground in liquid nitrogen, and metabolites were extracted in $4 \times$ volume of extraction solvent $\left(\mathrm{CH}_{3} \mathrm{CN} / \mathrm{H}_{2} \mathrm{O} / \mathrm{Hac}, 79: 20: 1\right)$. DON and D3G levels were ascertained by LC-MS/MS according to Vendl et al. (2009).

Field screening of transgenic lines against $F$. graminearum.

Field tests were conducted in the summers of 2012, 2013, and 2014 at the University of Minnesota Agricultural Experiment Station (UMore Park) in Rosemount, MN, U.S.A. Seeds for the transgenic events included in the field trials were obtained from ELISA-positive plants in the previous generation. Nontransformed controls (BW, CBO37, Rollag, Wheaton, and Sumai 3) were also planted. Entries were arranged in a randomized complete block design with four replications. All the lines were spray-inoculated twice. The first inoculation was applied at anthesis. The second inoculation was made 3 days after the initial application for each plot. The inoculum was a composite of 30 to $39 \mathrm{~F}$. graminearum isolates at a concentration of 100,000 macroconidia per milliliter. The inoculum was applied using a $\mathrm{CO}_{2}$-powered sprayer fitted with a SS8003 TeeJet spray nozzle with an output of $10 \mathrm{ml}$ per second, at a working pressure of $275 \mathrm{kPa}$. Mist-irrigation was applied from the first inoculation through disease assessment to facilitate FHB development. FHB incidence and severity were assessed visually 19 to 27 dai on 20 arbitrarily selected heads per plot. FHB incidence was determined by the percentage of spikes with visually symptomatic spikelets of the 20 heads observed. FHB severity was determined as the percent symptomatic spikelets of the total of all spikelets observed. Approximately 60 heads were hand harvested from each plot at maturity, were threshed, and the seeds were cleaned manually. The cleaned grain samples were then used to determine the percentage of VSK (Jones and Mirocha 1999). DON concentrations in the 2012 field trial was conducted by gas chromatography-MS (Fuentes et al. 2005). DON and D3G concentrations of the grain samples in the 2013 and 2014 trials were measured using LC-MS/MS (Vendl et al. 2009).

In the 2013 and 2014 field trials, plant height was measured in the $\mathrm{BW}$ and $\mathrm{CBO} 37$ transgenic plants and nontransformed controls.

\section{Root growth assay.}

Surface-sterilized wheat seeds of the nontransformed BW and CBO37 controls and transgenic events \#8, \#15, \#37, \#1381, \#1726, \#1386, and \#1383 expressing HvUGT13248 were plated on MS growth medium to germinate. One day after germination, seven seedlings of each genotype were transferred to a square petri dish with MS medium containing DON. For BW transgenic plants and the $\mathrm{BW}$ nontransformed control, the seedlings were plated on $0,2,5$, or $10 \mathrm{ppm} \mathrm{DON}$, and, for the CBO37 transgenic plants and CBO37 nontransformed control, the seedlings were plated on 0,2 , and $5 \mathrm{ppm}$ DON. Three replicate plates of each DON concentration were used. The 
plates were positioned vertically under white light at room temperature. Pictures were taken of the plates every day from 3 days after germination. Root growth of seedlings was determined by measuring the longest root of each seedling from the photos, using the ImageJ program (Rasband, W.S.; ImageJ, U. S. National Institutes of Health, Bethesda, Maryland, U.S.A.).

\section{ACKNOWLEDGMENTS}

We thank Y. Huang and A. Hofstad in the Muehlbauer lab for help and discussions. We also acknowledge Y. Dong for conducting the DON concentration analysis for samples from the field trials. We also thank A. M. Elakkad for field trial management. This work was supported by grants from the United States Department of Agriculture-Agrecultural Research Service U.S. Wheat and Barley Scab Initiative and the Minnesota Small Grains Initiative to G. J. Muehlbauer.

\section{LITERATURE CITED}

Anderson, J. A., Stack, R. W., Liu, S., Waldron, B. L., Fjeld, A. D., Coyne, C., Moreno-Sevilla, B., Fetch, J. M., Song, Q. J., Cregan, P. B., and Frohberg, R. C. 2001. DNA markers for Fusarium head blight resistance QTLs in two wheat populations. Theor. Appl. Genet. 102:1164-1168.

Bai, G. H., Desjardins, A. E., and Plattner, R. D. 2002. Deoxynivalenolnonproducing Fusarium graminearum causes initial infection, but does not cause disease spread in wheat spikes. Mycopathologia 153: 91-98.

Berthiller, F., Dall'asta, C., Corradini, R., Marchelli, R., Sulyok, M., Krska, R., Adam, G., and Schuhmacher, R. 2009. Occurrence of deoxynivalenol and its 3- $\beta$-D-glucoside in wheat and maize. Food Addit. Contam. Part A26:507-511.

Berthiller, F., Dall'Asta, C., Schuhmacher, R., Lemmens, M., Adam, G., and Krska, R. 2005. Masked mycotoxins: Determination of a deoxynivalenol glucoside in artificially and naturally contaminated wheat by liquid chromatography-tandem mass spectrometry. J. Agric. Food Chem. 53: 3421-3425.

Berthiller, F., Krska, R., Domig, K. J., Kneifel, W., Juge, N., Schuhmacher, R., and Adam, G. 2011. Hydrolytic fate of deoxynivalenol-3-glucoside during digestion. Toxicol. Lett. 206:264-267.

Bin-Umer, M. A., McLaughlin, J. E., Basu, D., McCormick, S., and Tumer, N. E. 2011. Trichothecene mycotoxins inhibit mitochondrial translation-Implication for the mechanism of toxicity. Toxins (Basel) 3:1484-1501.

Boddu, J., Cho, S., and Muehlbauer, G. J. 2007. Transcriptome analysis of trichothecene-induced gene expression in barley. Mol. Plant-Microbe Interact. 20:1364-1375.

Boenisch, M. J., and Schäfer, W. 2011. Fusarium graminearum forms mycotoxin producing infection structures on wheat. BMC Plant Biol. 11:110.

Brown, N. A., Urban, M., van de Meene, A. M. L., and Hammond-Kosack, K. E. 2010. The infection biology of Fusarium graminearum: Defining the pathways of spikelet to spikelet colonisation in wheat ears. Fungal Biol. 114:555-571.

Buerstmayr, H., Ban, T., and Anderson, J. 2009. QTL mapping and markerassisted selection for Fusarium head blight resistance in wheat: A review. Plant Breed. 128:1-26.

Caputi, L., Malnoy, M., Goremykin, V., Nikiforova, S., and Martens, S. 2012. A genome-wide phylogenetic reconstruction of family 1 UDPglycosyltransferases revealed the expansion of the family during the adaptation of plants to life on land. Plant J. 69:1030-1042.

Cheng, M., Fry, J. E., Pang, S., Zhou, H., Hironaka, C. M., Duncan, D. R., Conner, T. W., and Wan, Y. 1997. Genetic transformation of wheat mediated by Agrobacterium tumefaciens. Plant Physiol. 115: 971-980.

Cheng, W., Song, X.-S., Li, H.-P., Cao, L.-H., Sun, K., Qiu, X.-L., Xu, Y.-B., Yang, P., Huang, T., Zhang, J.-B., Qu, B., and Liao, Y.-C. 2015. Host-induced gene silencing of an essential chitin synthase gene confers durable resistance to Fusarium head blight and seedling blight in wheat Plant Biotechnol. J. Published online. doi:10.1111/pbi.12352.

Christensen, A. H., Sharrock, R. A., and Quail, P. H. 1992. Maize polyubiquitin genes: Structure, thermal perturbation of expression and transcript splicing, and promoter activity following transfer to protoplasts by electroporation. Plant Mol. Biol. 18:675-689.

Desjardins, A., Proctor, R., Bai, G., McCormick, S. P., Shaner, G., Buechley, G., and Hohn, T. M. 1996. Reduced virulence of trichothecene-nonproducing mutants of Gibberella zeae in wheat field tests. Mol. Plant-Microbe Interact. 9:775-781.
Di, R., Blechl, A., Dill-Macky, R., Tortora, A., and Tumer, N. E. 2010 Expression of a truncated form of yeast ribosomal protein L3 in transgenic wheat improves resistance to Fusarium head blight. Plant Sci. 178:374-380.

European Commission. Regulation No. 1881 of 19 December 2006. Setting maximum levels for certain contaminants in foodstuffs. Off. J. Eur Union L364:5-24

Fuentes, R. G., Mickelson, H. R., Busch, R. H., Dill-Macky, R., Evans, C. K., Thompson, W. G., Wiersma, J. V., Xie, W., Dong, Y., and Anderson, J. A 2005. Resource allocation and cultivar stability in breeding for Fusarium head blight resistance in spring wheat. Crop Sci. 45:1965-1972.

Gardiner, S. A., Boddu, J., Berthiller, F., Hametner, C., Stupar, R. M. Adam, G., and Muehlbauer, G. J. 2010. Transcriptome analysis of the barley-deoxynivalenol interaction: Evidence for a role of glutathione in deoxynivalenol detoxification. Mol. Plant-Microbe Interact. 23:962-976.

Goswami, R. S., and Kistler, H. C. 2004. Heading for disaster: Fusarium graminearum on cereal crops. Mol. Plant Pathol. 5:515-525.

Hajdukiewicz, P., Svab, Z., and Maliga, P. 1994. The small, versatile $p P Z P$ family of Agrobacterium binary vectors for plant transformation. Plant Mol. Biol. 25:989-994.

Himmelbach, A., Zierold, U., Hensel, G., Riechen, J., Douchkov, D., Schweizer, P., and Kumlehn, J. 2007. A set of modular binary vectors for transformation of cereals. Plant Physiol. 145:1192-1200.

Jansen, C., von Wettstein, D., Schäfer, W., Kogel, K.-H., Felk, A., and Maier, F. J. 2005. Infection patterns in barley and wheat spikes inoculated with wild-type and trichodiene synthase gene disrupted Fusarium graminearum. Proc. Natl. Acad. Sci. U.S.A. 102:16892 16897.

Jones, R. K., and Mirocha, C. J. 1999. Quality parameters in small grains from Minnesota affected by Fusarium head blight. Plant Dis. 83: 506-511.

Kazan, K., Gardiner, D. M., and Manners, J. M. 2012. On the trail of a cereal killer: Recent advances in Fusarium graminearum pathogenomics and host resistance. Mol. Plant Pathol. 13:399-413.

Lemmens, M., Scholz, U., Berthiller, F., Dall'Asta, C., Koutnik, A., Schuhmacher, R., Adam, G., Buerstmayr, H., Mesterházy, A., Krska, R., and Ruckenbauer, P. 2005. The ability to detoxify the mycotoxin deoxynivalenol colocalizes with a major quantitative trait locus for Fusarium head blight resistance in wheat. Mol. Plant-Microbe Interact. 18:1318-1324.

Li, Y., Baldauf, S., Lim, E. K., and Bowles, D. J. 2001. Phylogenetic analysis of the UDP-glycosyltransferase multigene family of Arabidopsis thaliana. J. Biol. Chem. 276:4338-4343.

Liu, S., Zhang, X., Pumphrey, M. O., Stack, R. W., Gill, B. S., and Anderson, J. A. 2006. Complex microcolinearity among wheat, rice, and barley revealed by fine mapping of the genomic region harboring a major QTL for resistance to Fusarium head blight in wheat. Funct. Integr. Genomics 6:83-89.

Lysøe, E., Seong, K.-Y., and Kistler, H. C. 2011. The transcriptome of Fusarium graminearum during the infection of wheat. Mol. Plant-Microbe Interact. 24:995-1000.

Mackintosh, C. A., Garvin, D. F., Radmer, L. E., Heinen, S. J., and Muehlbauer, G. J. 2006. A model wheat cultivar for transformation to improve resistance to Fusarium Head Blight. Plant Cell Rep. 25:313-319.

Mackintosh, C. A., Lewis, J., Radmer, L. E., Shin, S., Heinen, S. J., Smith, L. A., Wyckoff, M. N., Dill-Macky, R., Evans, C. K., Kravchenko, S., Baldridge, G. D., Zeyen, R. J., and Muehlbauer, G. J. 2007. Overexpression of defense response genes in transgenic wheat enhances resistance to Fusarium head blight. Plant Cell Rep. 26:479-488.

Masuda, D., Ishida, M., Yamaguchi, K., Yamaguchi, I., Kimura, M., and Nishiuchi, T. 2007. Phytotoxic effects of trichothecenes on the growth and morphology of Arabidopsis thaliana. J. Exp. Bot. 58:1617-1626.

McCormick, S. P., Alexander, N. J., Trapp, S. E., and Hohn, T. M. 1999. Disruption of TRI101, the gene encoding trichothecene 3-Oacetyltransferase, from Fusarium sporotrichioides. Appl. Environ. Microbiol. 65:5252-5256.

Mesterhazy, A. 1995. Types and components of resistance to Fusarium head blight of wheat. Plant Breed. 114:377-386.

Muhitch, M. J., McCormick, S. P., Alexander, N. J. N., and Hohn, T. M. 2000. Transgenic expression of the TRI101 or PDR5 gene increases resistance of tobacco to the phytotoxic effects of the trichothecene 4,15diacetoxyscirpenol. Plant Sci. 157:201-207.

Okubara, P. A., Blechl, A. E., McCormick, S. P., Alexander, N. J., DillMacky, R., and Hohn, T. M. 2002. Engineering deoxynivalenol metabolism in wheat through the expression of a fungal trichothecene acetyltransferase gene. Theor. Appl. Genet. 106:74-83.

Parry, D. W., Jenkinson, P., and McLeod, L. 1995. Fusarium ear blight (scab) in small-grain cereals-A review. Plant Pathol. 44:207-238.

Pestka, J. J. 2010. Deoxynivalenol: Mechanisms of action, human exposure, and toxicological relevance. Arch. Toxicol. 84:663-679. 
Poppenberger, B., Berthiller, F., Lucyshyn, D., Sieberer, T., Schuhmacher, R., Krska, R., Kuchler, K., Glössl, J., Luschnig, C., and Adam, G. 2003. Detoxification of the Fusarium mycotoxin deoxynivalenol by a UDPglucosyltransferase from Arabidopsis thaliana. J. Biol. Chem. 278: 47905-47914.

Poppenberger, B., Fujioka, S., Soeno, K., George, G. L., Vaistij, F. E., Hiranuma, S., Seto, H., Takatsuto, S., Adam, G., Yoshida, S., and Bowles, D. 2005. The UGT73C5 of Arabidopsis thaliana glucosylates brassinosteroids. Proc. Natl. Acad. Sci. U.S.A. 102:15253-15258.

Proctor, R. H., Hohn, T. M., and McCormick, S. P. 1995. Reduced virulence of Gibberella zeae caused by disruption of a trichothecene toxin biosynthetic gene. Mol. Plant-Microbe Interact. 8:593-601.

Proctor, R. H., Hohn, T. M., and McCormick, S. P. 1997. Restoration of wildtype virulence to Tri5 disruption mutants of Gibberella zeae via gene reversion and mutant complementation. Microbiology 143:2583-2591.

Ross, J., Li, Y., Lim, E., and Bowles, D. J. 2001. Higher plant glycosyltransferases. Genome Biol. 2:reviews3004.1-reviews3004.6.

Schweiger, W., Boddu, J., Shin, S., Poppenberger, B., Berthiller, F., Lemmens, M., Muehlbauer, G. J., and Adam, G. 2010. Validation of a candidate deoxynivalenol-inactivating UDP-glucosyltransferase from barley by heterologous expression in yeast. Mol. Plant-Microbe Interact. 23:977-986.

Schweiger, W., Pasquet, J.-C., Nussbaumer, T., Paris, M. P. K., Wiesenberger, G., Macadré, C., Ametz, C., Berthiller, F., Lemmens, M., Saindrenan, P., Mewes, H.-W., Mayer, K. F. X., Dufresne, M., and Adam, G. 2013.
Functional characterization of two clusters of Brachypodium distachyon UDP-glycosyltransferases encoding putative deoxynivalenol detoxification genes. Mol. Plant-Microbe Interact. 26:781-792.

Shin, S., Mackintosh, C. A., Lewis, J., Heinen, S. J., Radmer, L., DillMacky, R., Baldridge, G. D., Zeyen, R. J., and Muehlbauer, G. J. 2008 Transgenic wheat expressing a barley class II chitinase gene has enhanced resistance against Fusarium graminearum. J. Exp. Bot. 59: 2371-2378.

Shin, S., Torres-Acosta, J. A., Heinen, S. J., McCormick, S., Lemmens, M., Paris, M. P. K., Berthiller, F., Adam, G., and Muehlbauer, G. J. 2012 Transgenic Arabidopsis thaliana expressing a barley UDP-glucosyltransferase exhibit resistance to the mycotoxin deoxynivalenol. J. Exp. Bot. 63 : 4731-4740.

United States Food and Drug Administration. 2010. Guidance for industry and FDA: Advisory levels for deoxynivalenol (DON) in finished wheat products for human consumption and grains and grain by-products used for animal feed. United States Food and Drug Administration, Silver Spring, MD, U.S.A.

Vendl, O., Berthiller, F., Crews, C., and Krska, R. 2009. Simultaneous determination of deoxynivalenol, zearalenone, and their major masked metabolites in cereal-based food by LC-MS-MS. Anal. Bioanal. Chem. 395:1347-1354.

Waldron, B., Moreno-Sevilla, B., and Anderson, J. 1999. RFLP mapping of QTL for Fusarium head blight resistance in wheat. Crop Sci. 39: 805-811. 